\title{
Regina-Maria Dackweiler
}

\section{Staatlichkeit, Gewalt und Geschlecht: Bekämpfung von (sexueller) Gewalt im sozialen Nahbereich als Staatsaufgabe}

\section{Einleitung}

Misshandlung und sexuelle Gewalt gegen Frauen und Mädchen im sozialen Nahbereich gelten mittlerweile als ein Politikum und als ein vom Staat zu bearbeitendes Problem. Nicht mehr als individuelle Schande verdrängt und persönliches Schicksal verharmlost, sondern als Menschenrechtsverletzung definiert, wird diese Gewalt, ausgehend von Ehemännern, Partnern, Vätern und Verwandten, unterdessen gesellschaftlich verurteilt und rechtsstaatlich geahndet. Für die übernommene staatliche Verantwortung des Schutzes und der Unterstützung der Gewaltopfer sowie der Inverantwortungnahme der Gewalttäter kommen zentrale Interventionsmedien wie Zwangsgewalt, Recht, Geld und Wissen zum Einsatz. Dies sollte entsprechend der viel zitierten Definition des modernen Staates durch den liberalen Nationalökonomen und Soziologen, Max Weber, ${ }^{1}$ als derjenigen „menschlichen Gemeinschaft, welche innerhalb eines bestimmten Gebietes [...] das Monopol legitimer physischer Gewaltsamkeit für sich (mit Erfolg) beansprucht“, auch nicht bemerkenswert sein. Angesichts der jahrzehntelangen Untätigkeit des bundesdeutschen Staates gegenüber dieser „ganz gewöhnlichen Gewalt" 2 hinter den geschlossenen Türen der Institutionen von Ehe und Familie ist die Fülle der Verrechtlichungen und Aktivitäten der letzten Jahre jedoch durchaus erklärungsbedürftig. Denn trotz des vorausgesetzten physischen Gewaltmonopols des sozialstaatlich flankierten bundesdeutschen Rechtsstaates beruhen alle genannten Interventionsmedien zur (sexuellen) Gewalt gegen Frauen im sozialen Nahbereich auf einer gerade einmal seit einer Dekade konsensualen Neudefinition dessen, was zu den Staatsaufgaben zählt, die - wie an diesem geschlechterpolitisch hochrelevanten Beispiel überdeutlich zu erkennen ist - jeweils anerkannt, durchgesetzt, institutionalisiert und verteidigt werden müssen. ${ }^{3}$

So lässt sich die in der Bundesrepublik Deutschland erst in der zweiten Hälfte der 1990er Jahre verbindlich gewordene Neudefinition der staatlichen Agenda in Bezug auf Gewalt im Geschlechterverhältnis als Ergebnis langjähriger und zäher politischer Auseinandersetzungen und Kämpfe um die Legitimation und Delegitimierung staatlicher Nichtintervention gegenüber der im „Privatbereich“ ausgeübten Gewalt gegen Frauen nachvollziehen. Warum aber erweist sich die Einhaltung bzw. Durchsetzung des staatlichen Gewaltmonopols im Falle der Gewalt gegen Frauen im sozialen Nahbereich als etwas, das erst „gewollt“ werden musste? Gegen welchen Widerstand galt es, diese so selbstverständlich erscheinende Staatsaufgabe zur Anerkennung zu bringen? Wer ist an diesen (geschlech-

1 Weber, Gesammelte politische Schriften, 1971, S. 506.

2 Benard/Schlaffer, Die ganz gewöhnliche Gewalt in der Ehe. Texte zu einer Soziologie von Macht und Liebe, 1978.

3 Offe, Die Aufgaben von staatlichen Aufgaben: „Thatcherismus“ und die populistische Kritik der Staatstätigkeit, in: Grimm (Hrsg.), Staatsaufgaben, 1996, S. 319. 
ter-)politischen Auseinandersetzungen und Kämpfen um die Durchsetzung der staatlichen „Monopolorganisation der Gewalttat [...] als einer Kontrollorganisation für das Verhalten des Einzelnen "4 an welchen Orten, in welcher Form und mit welchen Motiven beteiligt? Welche Ressourcen kamen unter welchen Handlungsbedingungen in diesen Kämpfen zum Einsatz, die eine Institutionalisierung staatlicher Interventionen vorantrieben? Und gegen wen und/oder was gilt es, die unterdessen gesetzte Agenda gegebenenfalls zu verteidigen?

Alle diese Fragen stecken den Umgang des bundesdeutschen Staats mit geschlechtsbezogener Gewalt im Konflikt mit frauen(-bewegungs-)politischen Akteurinnen und deren Ringen für eine Ent-Privatisierung und Neudefinition dieser Gewalt in einem macht- und herrschaftskritischen Horizont als ein exemplarisches staatstheoretisches Untersuchungsfeld ab. Im Folgenden soll aufgezeigt werden, dass und wie staatliche Politiken, d.h. staatliches Handeln und Nicht-Handeln, sowohl zur Ausgestaltung hierarchischer und gewaltförmiger Geschlechterverhältnisse beitragen als auch diese Verhältnisse als egalitäre und gewaltfreie zu normieren und sanktionieren vermögen. Hierbei verfolge ich die These, dass es sich beim Staat um ein Ensemble widersprüchlicher diskursiver Arenen und Institutionen handelt, in dem herrschaftsförmige Geschlechterverhältnisse, interferierend mit anderen hierachisierenden Sozialverhältnissen (insbesondere entlang der Kategorien Klasse, Hautfarbe, Ethnie und Nationalität), historisch geronnen sind. In diesen Diskursarenen sowie Institutionen der Staatsbürokratie, die als vergeschlechtlichte Strukturen und Kontexte in den Blick genommen werden können, versuchen interessengeleitete AkteurInnen hierarchische Geschlechterverhältnisse in ihrer Verschränkung mit einer heteronormativen symbolischen Ordnung der Zweigeschlechtlichkeit zu reproduzieren, zu modifizieren oder zu transformieren, wobei sie mit unterschiedlicher Verhandlungsmacht innerhalb und gegenüber den Staatsapparaten bzw. ungleichen $\mathrm{Zu}$ gängen zu deren Machtressourcen ausgestattet sind.

Zur Plausibilisierung dieser These werfe ich in einem ersten Schritt ein Schlaglicht auf die feministische Erfolgsgeschichte der Forderungen nach Enttabuisierung, Entprivatisierung und Entindividualisierung der Gewalt gegen Frauen im sozialen Nahbereich auf internationaler, supranationaler und nationaler politischer Ebene. In einem zweiten Schritt skizziere ich zentrale Dimensionen einer feministisch informierten Staatskonzeption, indem ich produktive Anknüpfungspunkte an kritisch-materialistische und diskurstheoretische Auffassungen von Staatlichkeit herausarbeite, die eine Analyse entlang der aufgeworfenen Fragen für dieses Untersuchungsfeld eröffnen. Den Anteil des Staates an der Herrschaftsförmigkeit der Geschlechterverhältnisse verdeutlicht - wie in keinem anderen Bereich geschlechterpolitischer Nicht-Intervention des Staates - die bis 1997 bestehende Straffreiheit gegenüber dem sexuell gewalttätigen Ehemann, verzichtete doch der Staat im entsprechenden Strafrechtsbereich auf das Gewaltmonopol, in dem er ehemännliche Verfügungsrechte über den weiblichen Körper unter dem Signum der „Privatsphäre“ kodifizierte und legitimierte. Daher wird im dritten Schritt empirisches Material über den drei Dekaden umfassenden Prozess der Verrechtlichung von Vergewaltigung in der Ehe als Straftatbestand gegen die sexuelle Selbstbestimmung entfaltet und im exemplarische Sinne auf diese Gewaltform gegen Frauen fokussiert. In einem vierten Schritt werde ich die skizzierte staatstheoretische Konzeption für das entfaltete empirische Untersuchungsfeld zur Anwendung bringen und das analytische Potential der Konzeption ausloten. Im Fazit begründe ich den von mir als ambivalent bewerteten 
Konsens über die neue Staatsaufgabe des Schutzes der Menschen- und Grundrechte von Frauen im Fall von (sexualisierter) Gewalt im sozialen Nahbereich.

\section{Schutz der Opfer und Inverantwortungnabme der Täter: Bekämpfung (sexueller) Gewalt im sozialen Nabbereich als Staatsaufgabe}

Seit annähernd zwei Dekaden lässt sich eine wachsende weltweite Anerkennung gegenüber Frauenrechten beobachten sowie ein international gestiegenes Interesse an der Gleichstellung von Frauen auf politischer, sozialer, ökonomischer und kultureller Ebene. ${ }^{5}$ Seit der UN-Menschenrechtskonferenz in Wien 1993 kann man von einem internationalen Konsens bezüglich der Rede von „Frauen sind Menschenrechte“ sprechen - ein Konsens, der Anliegen der Entwicklung mit denen der Menschenrechte verbindet und das Problem der Misshandlung von und sexualisierten Gewalt gegen Frauen und Mädchen einschließt.

In diesem globalen „window of opportunity“ gelang es Dank der langjährigen Aktivitäten der transnational vernetzt agierenden Frauenbewegungen ${ }^{6}$ auf internationaler Ebene der Vereinten Nationen zunächst, zwei „General Recommendations on Violence against women“, Nr. 12 und Nr. 19, in den Jahren 1989 bzw. 1992 zur Verabschiedung zu bringen. Hierbei handelt es sich um Empfehlungen zur Auslegung und Realisierung der Frauenrechtskonvention „CEDAW“, dem „Übereinkommen zur Beseitigung jeder Form von Diskriminierung der Frau“ aus dem Jahr 1979, welche die Vertragsstaaten bei Maßnahmen zur Unterbindung von Frauenfeindlichkeit und Gewalthandlungen auch von als privat definierter „häuslicher Gewalt“ unterstützen sollen, thematisiert doch die „Magna Charta“ der Menschenrechte von Frauen ebenso wenig explizit geschlechtsbezogene Gewalt in ihren 30 Artikeln wie die „Allgemeine Erklärung der Menschenrechte“. 1993 verabschiedete die UN die „Deklaration über die Beseitigung der Gewalt gegen Frauen“, die besagt, dass für die Beendigung von Gewalt gegen Frauen als Menschenrechtsverletzung die Staaten mitverantwortlich sind, unabhängig davon, ob diese Gewalt im Öffentlichen oder Privaten geschieht. Die ein Jahr später durch die Generalversammlung bestellte erste UN-„Sonderberichterstatterin über Gewalt gegen Frauen, deren Gründe und Auswirkungen“, Rhadika Coormaraswamy, ${ }^{7}$ bezeichnete in ihrem Bericht „die Untätigkeit der Regierungen als wahrscheinlich wichtigste Ursache für Gewalt gegen Frauen“. Nicht zuletzt auf Grund der hohen weltweiten medialen Resonanz kommt auch dem in der „Aktionsplattform“ der Vierten Weltfrauenkonferenz in Beijing aus dem Jahr 1995 formulierten Abschnitt zum Problem der „Gewalt gegen Frauen“ herausragende symbolische Bedeutung zu, auch wenn der in diesem Abschlussdokument verfasste Handlungsplan völkerrechtlich nicht bindend ist, sondern nur Empfehlungscharakter hat.

Auch der Europarat engagierte sich zu diesem von ihm als entscheidend eingeschätzten Menschenrechtsthema und realisierte zunächst zwischen 2007 und 2008 eine Kampagne zur Beendigung der Gewalt gegen Frauen. Sodann wurde im Mai 2011 in Istanbul die über Jahre vorbereitete „Konvention des Europarates zur Verhütung und Bekämpfung von Gewalt gegen Frauen und häuslicher Gewalt“ als völkerrechtlich bindender Vertrag von bislang 13 Mitglied-

5 Tripp, The Evolution of Transnational Feminisms: Consenus, Conflict and New Dynamics, in: Ferree/ Tripp (Hrsg.), Global Feminism, 2006, S. 51.

6 Dackweiler, Frauenrechte sind Menschenrechte: Transnationale Frauenbewegungspolitik zwischen Erfolgsgeschichte und Rückschlägen, Zeitschrift für Menschenrechte, H. 1/2009.

7 Coormaraswamy, Vorläufiger Bericht der Sonderberichterstatterin zum Thema „Gewalt gegen Frauen Ursachen und Folgen“, in: Bundesministerium für Familie, Senioren, Frauen und Jugend, Materialien zur Frauenpolitik 45/1995, S. 16 . 
staaten (neben u.a. der Bundesrepublik Deutschland auch der Türkei) unterzeichnet, die sich hiermit auch zur Einrichtung von Telefon-Hotlines, Frauenhäusern, Beratungsstellen und besonderen medizinischen Diensten verpflichten. ${ }^{8}$

Auf supranationaler Ebene der EU wurden nicht nur mit den wegweisenden Programmen DAPHNE I. bis III. zwischen 2000 und 2011 finanzielle Mittel für innovative Maßnahmen nichtstaatlicher Organisationen, für Forschungsarbeiten, Kooperations- und Pilotprojekte im Kampf gegen geschlechtsbezogene Gewalt in der Gesamthöhe von rund 110 Millionen Euro bereit gestellt. Diese Programme, unterdessen angesiedelt in dem Allgemeinen Programm „Fundamental Rights and Justice“, ermöglichten auch den Fortbestand und die Weiterentwicklung der Arbeit des 1994 gegründeten internationalen feministischen Netzwerkes WAVE (Women against Violence Europe) mit Sitz in Wien, bestehend aus NGOs, die sich gegen Gewalt gegen Frauen im sozialen Nahbereich engagieren. Dieses Netzwerk wiederum beriet den Europarat bei dessen Kampagne und war als Expertinnen-Gruppe an der Ausformulierung der Europarat-Konvention beteiligt. Darüber hinaus formulierte der „Rat für Allgemeine Angelegenheiten“ im Dezember 2008 die „Leitlinien der EU betreffend Gewalt gegen Frauen und die Bekämpfung aller Formen der Diskriminierung von Frauen“: Zu deren operativen Zielen gehört die Bekämpfung der Straflosigkeit der Gewalttäter, indem Gewalt gegen Frauen rechtlich geahndet und Maßnahmen ergriffen werden, die den Opfern den Zugang zur Justiz erleichtern.

Auf nationaler Ebene zeugen vielfältige Aktivitäten davon, dass der bundesdeutsche Staat sich den Schutz von Frauen vor Gewalt in der als privat geltenden Sphäre von Ehe, Familie und intimen Paarbeziehungen, auch im Vollzug der von ihr unterzeichneten Menschenrechtsdokumente, seit der zweiten Hälfte der 1990er Jahren zur Aufgabe gemacht hat. Zu nennen sind der erste und zweite „Aktionsplan der Bundesregierung zur Bekämpfung von Gewalt gegen Frauen“, ${ }^{9}$ die Schaffung von „Interventionsprojekten gegen häusliche Gewalt", von Sonderdezernaten der Staatsanwaltschaft, Projekten zur Arbeit mit gewalttätigen Männern sowie die Einrichtung einer „Bund-Länder-Arbeitsgruppe“ unter Federführung des Bundesministeriums für Familie, Senioren, Frauen und Jugend (BMFSFJ) zu diesem Thema, bestehend aus Bundes- und Länderministerien, dem Bundeskriminalamt und frauenpolitischen Nicht-Regierungsorganisationen. Vergewaltigung in der Ehe wurde im Jahr 1997 erstmals als Straftatbestand normiert. Mit Bezug auf sexuelle Gewalt (in der Ehe) wurden Fortbildungsangebote für die Strafverfolgungsbehörden sowie Fachkommissariate und Sonderstaatsanwaltschaften geschaffen, um die spezifischen Bedürfnisse der Opfer wahrzunehmen und deren erneute Viktimisierung zu verhindern. Januar 2002 trat - flankiert von reformierten Polizeigesetzen der Länder zur Gefahrenabwehr bei „häuslicher Gewalt“ - das „Gesetz zum zivilrechtlichen Schutz vor Gewalttaten und Nachstellungen“ in Kraft sowie der hier verankerte Anspruch auf Überlassen der gemeinsam genutzten Wohnung für das Opfer unter dem Motto „Wer schlägt, der geht“. Sodann wurde angesichts der besonderen rechtlichen und sozialen Not von Migrantinnen eine Fristverkürzung für ein eigenständiges Aufenthaltsrecht nachgezogener Familienangehöriger von vier auf zwei Jahre, bei Härtefällen auf ein Jahr, realisiert. Der bundesdeutsche Staat engagiert sich in der Ko-Finanzierung bundesweiter Netzwerke von Frauenhäusern, -bera-

8 Europarat, http://conventions.coe.int/Treaty/EN/Treaties/Html/210.htm (abgerufen am 18. Dezember 2011).

9 BMFSJ, Aktionsplan der Bundesregierung zur Bekämpfung von Gewalt gegen Frauen, 1999; BMFSJ, Aktionsplan II zur Bekämpfung von Gewalt gegen Frauen, 200\%? 
tungsstellen und -notrufen und unterstützt deren Öffentlichkeitskampagnen für ein „gewaltfreies Leben von Frauen“, und er vergibt Forschungsaufträge, so etwa zur Prävalenz von Gewalt gegen Frauen sowie zur Evaluation der geschaffenen Interventionsprojekte. ${ }^{10}$ Auch in den Staatsapparaten selbst wurde das Thema institutionalisiert, besteht doch im BMFSFJ in der Unterabteilung „Gleichstellung“ seit einigen Jahren ein eigenständiges Referat zum „Schutz von Frauen vor Gewalt".

Fasst man diese Höhepunkte des bis heute Erreichten im Kampf gegen Misshandlung und sexuelle Gewalt gegen Frauen im sozialen Nahbereich zusammen, liest sich dies wie eine beeindruckende Erfolgsgeschichte der Kodifizierung von Rechten und Ansprüchen für die von geschlechtsbezogener Gewalt Betroffenen auf allen politischen Ebenen staatlichen Handelns, konkret nationaler, internationaler und supranationaler. Deutlich zu machen gilt jedoch, dass es sich um eine Geschichte handelt, deren sichtbare Ergebnisse nicht zu denken sind ohne die aufmerksamkeitsstarken Aktivitäten und Kampagnen, (Selbsthilfe-)Projekte und wissenschaftlichen Forschungsarbeiten der Neuen Frauenbewegungen seit den 1970er Jahren im Kampf gegen Tabuisierung, Normalisierung und Legitimierung dieser Gewaltformen durch die verschiedenen Staatsapparate. Dessen gewärtig, zeigt sich ein doppelter blinder Fleck staatstheoretischer Analysen: Weder befasst sich die deutschsprachige staatstheoretische Diskussion unterschiedlichster Ausrichtungen bislang mit dem Zusammenhang von geschlechtsbezogener Gewalt in Ehe und Familie und staatlicher Politik noch hat die feministisch orientierte Politikwissenschaft diesen Zusammenhang aus einer staatstheoretischen Perspektive bislang hinreichend systematisch aufgegriffen. ${ }^{11} \mathrm{Im}$ folgenden Abschnitt sollen daher Dimensionen einer feministisch informierten Staatskonzeption vorgestellt werden, die eine solche Perspektive einzunehmen vermögen.

\section{Geschlechtsspezifische Selektivitäten des Staates und dessen Subjektivierungsmacht}

Feministische Forschung benannte zwar von Beginn an die moderne Sphärentrennung als Legitimationsfolie der Tabuisierung und Nicht-Ahndung geschlechtsbezogener Gewalt im sozialen Nahbereich. Konkret verwies sie auf die geschlechterpolitischen Konsequenzen der Trennung von „Öffentlichem“, sprich die entlang von Warenproduktion, Konkurrenz, Macht und struktureller Gewalt organisierten gesellschaftlichen Bereiche von Ökonomie und Politik einerseits und dem an Gebrauchswertproduktion, Konsens, Reziprozität und Liebe in der Intimität von Ehe und Familie orientierten „Privatem“ andererseits. Zugleich besteht vor dem Hintergrund der erfolgreichen Mobilisierung der transnational agierenden Frauenbewegungen des globalen Südens und Nordens für die Anerkennung von (sexualisierter) Gewalt gegen Frauen im sozialen Nahbereich als Menschenrechtsverletzung unterdessen weitestgehend Konsens darüber, dass sexualisierte Gewalt und Misshandlung als „Praktiken der Mikro-

10 Müller, Gewalt: Von der Enttabuisierung zur Einfluss nehmenden Forschung, in: Becker/Kortendieck (Hrsg.), Handbuch der Frauen- und Geschlechterforschung. Theorie, Methoden, Empirie, 2008, S. $660-668$.

11 Erste systematische Annäherungen in diese Richtung wurden vorgelegt in Dackweiler/Schäfer (Hrsg.), Gewalt-Verhältnisse. Feministische Perspektiven auf Geschlecht und Gewalt, 2002, sowie in Ludwig/ Sauer/Wöhl (Hrsg.), Staat und Geschlecht. Grundlagen und aktuelle Herausforderungen feministischer Staatstheorie, 2009. 
Interaktion und des körperlichen Habitus"12 zur Aufrechterhaltung einer herrschaftsförmigen Geschlechterordnung nur dann verstanden werden können, wenn diese „privaten“ Praktiken nicht nur politisiert, sondern zugleich mit Blick auf das Eingreifen bzw. Nichteingreifen des Staates analysiert werden. ${ }^{13}$ Jedoch erweisen sich zur Beantwortung der hier aufgeworfenen Fragen aus dieser Perspektive liberale Staatstheorietraditionen nicht als zweckmäßig. Denn diese betrachten den Staat als einen geschlechtsneutralen Schiedsrichter, der vermeintlich nicht nur über den Konflikten der Markt- als Konkurrenzgesellschaft, sondern auch über jenen der gewaltförmigen Verhältnisse der Geschlechter in der bürgerlichen Privatheit von Ehe und Familie steht - Verhältnisse, die er ja bis in die jüngste Vergangenheit gerade nicht zu dämpfen und im Rekurs auf allgemeinverbindliche Regelungen, sprich Recht und dessen Durchsetzung, zu befrieden suchte. Noch erscheint - in Analogie zu Strängen marxistischer Theorie des Staates als eines ideellen „Gesamtkapitalisten“ - dessen Konzeption als ein ideeller „Gesamtpatriarch“ geeignet, um die Orte und Logiken der Konflikte und Kämpfe sowie die Dynamik der Allianzen und Kompromisse der AkteurInnen für und gegen eine Transformation gewaltförmiger Geschlechterverhältnisse zu erklären.

Demgegenüber stellt sich die „strategisch-relationale Perspektive“ des neo-marxistischen Staatstheoretikers Bob Jessop ${ }^{14}$ als weiterführend dar, mit der er beabsichtigt, den Dualismus von Struktur und Handlungsfähigkeit in Kapitalismus- und Klassentheorien für eine Theorie des komplexen Verhältnisses von Wirtschaft und Staat zu überwinden - eine Perspektive, die er explizit auch für eine „alternative Betrachtungsweise des Verhältnisses zwischen patriarchalen Strukturen und Handlungen vergeschlechtlichter Subjekte“ fruchtbar machen will, indem er „geschlechtsspezifische Selektivitäten “15 in den analytischen Fokus zu rücken versucht. Weil es für Jessop gilt, Strukturen auf die ihnen eingeschriebenen „strategischen Selektivitäten“ und Handlungen auf ihre unterschiedlich reflexive „strukturorientierte strategische Kalkulation“ zu untersuchen, öffnet er den Fragenhorizont nach der möglichen Privilegierung bestimmter Identitäten, Interessen und Strategien im Staat und danach, „auf welche Weise (individuelle und/oder kollektive) AkteurInnen diese unterschiedliche Privilegierung unter Umständen bei der Wahl ihrer Vorgehensweise im Rahmen von strategischen Kontextanalysen berücksichtigen “. ${ }^{16}$ Jessops Konzeption ist somit für das hier fokussierte Untersuchungsfeld der Auseinandersetzungen über eine Verrechtlichung von Vergewaltigung in der Ehe anschlussfähig, verspricht er doch einen analytischen Zugang zur Dialektik von patriarchalen Strukturendimensionen staatlicher Apparate, in deren Form und Inhalt Strategien geronnen sind, und den strukturell orientierten Praktiken interessengeleiteter, staatlicher und nichtstaatlicher AkteurInnen innerhalb und außerhalb dieser Apparate.

Für seine Konzeptualisierung der (geschlechtsspezifischen) strategischen Selektivitäten des Staates rekurriert Jessop erstens auf die in den 1920 er Jahren formulierten Überlegungen von Antonio Gramsci ${ }^{17}$ zum „integralen Staat“. Dieser besteht nach Gramsci zum einen aus der „politischen Gesellschaft“, d.h. Parteien,

Klinger, Macht - Herrschaft - Gewalt, in: Rosenberger/Sauer (Hrsg.), Politikwissenschaft und Geschlecht, 2004, S. 103.

13 Kavemann/Grieger, Interventionsprojekte zur „Entprivatisierung“ der häuslichen Gewalt, in: Heitmeyer,/Schröttle(Hrsg.), Gewalt. Bonn (Bundeszentrale für politische Bildung) 2006, S. 125.

14 Jessop, Die geschlechtsspezifischen Selektivitäten des Staates, in: Kreisky/Lang/Sauer (Hrsg.), EU. Geschlecht. Staat, Wien 2001, S. 55.

15 Ebd.

16 Ebd. 56.

17 Gramsci, Gefängnishefte. Kritische Gesamtausgabe, hrsgg. v. Bochmann/Haug, $1991 \mathrm{ff}$. 
Verbände, Militär, Polizei, Justiz, Staatsbürokratie, und zum anderen aus der „Zivilgesellschaft“, u.a. Vereine, Clubs, Bildungssystem, Medien, Wissenschaft, Kirchen sowie Kunst- und Kultureinrichtungen. Während der Staat in der „politischen Gesellschaft“ seine direkte Herrschaft und formelle Regierung mit Recht, Zwang und Gewalt realisiere, entfalte der Staat in der "Zivilgesellschaft“ seine Macht über Prozesse der Führung, Willensbildung und die Schaffung von Allianzen durch geteilte Überzeugungen, Diskussionen, Verhandlungen und Kompromisse, was Gramsci als Hegemonie bezeichnet. ${ }^{18}$ Gramsci erweitert also den Staat um jene gesellschaftliche Sphäre, die von liberalen Staatstheoretikern zwischen der kapitalistischen Ökonomie zur einen und der Instanz der Recht setzenden und Gewalt ausübenden Staatsmacht zur anderen Seite als das Ensemble der „gemeinhin ,privat' genannten Organismen “19 situiert wird. Er weist somit die Grenzziehung zwischen „privat“, sprich: partikular, apolitisch und selbstregulierend, und „öffentlich“, sprich: universal, politisch und öffentlicher Intervention unterworfen, zurück, die seit den von Thomas Hobbes und John Locke formulierten liberalen politischen Theorien des "Gesellschaftsvertrages“ zwischen bürgerlicher Gesellschaft und Staat staatsteoretisch festgeschrieben wurde. Somit gelingt es nach Gramsci dem „integralen Staat“, die Konflikte und Kämpfe zwischen Herrschenden und Beherrschten darüber, was in Bezug auf die gesellschaftlichen Antagonismen der kapitalistischen Produktionsverhältnisse als privat und was als öffentlich prozessiert wird, aus der „politischen“ in die „zivile Gesellschaft“ zu verschieben, wo entsprechend deren spezifischer Logik diese Konflikte von (kollektiven) Akteuren mit den ihnen jeweils verfügbaren Mitteln in langwierigen Auseinandersetzungen bearbeitet würden. Hier gehe es sowohl um die Herstellung von Konsens zwischen den Fraktionen der herrschenden Klasse als auch um die Erlangung von Zustimmung der herrschaftsunterworfenen sozialen Gruppen und Klassen, die sich durch Kompromisse und Zugeständnisse herausbilde. ${ }^{20}$

Zweitens rekurriert Jessop auf den neo-marxistischen Staatstheoretiker Nicos Poulantzas, der in Orientierung an Gramsci dem Staat die Aufgabe zuschreibt, die Hegemonie der herrschenden Klasse aufrechtzuerhalten. Nach Poulantzas darf der Staat nicht als ein in sich abgeschlossenes Wesen begriffen werden, „sondern, wie auch das ,Kapital', als ein Verhältnis, genauer als die materielle Verdichtung eines Kräfteverhältnisses zwischen Klassen und Klassenfraktionen, das sich im Staat immer in spezifischer Form ausdrückt”. ${ }^{21}$ Doch geht er davon aus, dass der Staat über eine eigene Dichte und Widerstandskraft verfügt und sich nicht auf ein Kräfteverhältnis reduzieren lasse, er vielmehr als „relativ autonom” gegenüber den Produktionsverhältnisses zu betrachten sei. Daher unterscheidet Poulantzas einerseits zwischen „Staatsmacht“ als der Verdichtung eines Kräfteverhältnisses, das den Staat durchzieht und zugleich von ihm geformt wird, und andererseits - in Anlehnung an Louis Althusser ${ }^{22}$ - den „Staatsapparaten“, konkret dem repressiven Staatsapparat und den ideologischen Staatsapparaten, als der institutionellen Materialisierung dieses Kräfteverhältnisses. Die Staatsmacht

18 So formulierte Gramsci in einer für sein Denken über den „integralen Staat“ zentralen Notiz: „In dem Sinne könnte man sagen, daß Staat = politische Gesellschaft + Zivilgesellschaft, das heißt, Hegemonie, gepanzert mit Zwang“ (ebd., H. 6 \$88, S. 783).

19 Ebd., H. $12 \$ 1$, S. 1502.

20 Demirovic, Politische Gesellschaft - zivile Gesellschaft. Zur Theorie des integralen Staates bei Antonio Gramsci, in: Buckel/Fischer-Lescano (Hrsg.), Hegemonie gepanzert mit Zwang. Zivilgesellschaft und Politik im Staatsverständnis von Antonio Gramsci, 2007, S. 27.

21 Poulantzas, Staatstheorie, 2002, S. 159.

22 Althusser, Ideologie und ideologische Staatsapparate, 1977 
umfasst nach Poulantzas alle gesellschaftlichen Kräfte, die im und durch den Staat

agieren, doch sind nicht alle diese Kräfte in derselben Stärke präsent.

Hier ansetzend formuliert Jessop die Überlegung, dass das staatliche institutionelle Ensemble ungleich auf die Fähigkeit einzelner sozialer Kräfte einwirkt, bestimmte Interessen und Strategien innerhalb des Staates bzw. durch die Nutzung staatlicher Handlungsfähigkeit zu verfolgen: Der Staat biete bestimmten Gruppen mehr und anderen geringere Chancen, ihre Interessen zu artikulieren und zu organisieren. Auch in Bezug auf die Geschlechterverhältnisse muss nach Jessop der moderne, formal rationale Staat als strategisch-selektiv konstituiert betrachtet werden, der „mittels ,struktureller Kopplung' mit den anderen institutionellen Ordnungen der Gesellschaft verzahnt ist" ${ }^{23}$ Von Interesse sind sodann nicht nur die geschlechtsspezifisch strategisch-selektiven „Machtstrukturen“, sondern zugleich die „Politik der Macht“, ${ }^{24}$ d.h. die an jenen orientierten, strategischen Kalkulationen geschlechterpolitischer AkteurInnen, die mit Hilfe ihrer Kämpfe, Allianzen und Kompromisse diese Machtstrukturen zu transformieren suchen. Dies richtet die Aufmerksamkeit auf die Analyse eines Paradoxons, mit welchem sich Frauen konfrontiert sehen, die innerhalb des integralen Staates, also sowohl in der politischen als auch der zivilen Gesellschaft, gegen das geschlechterpolitische Handeln bzw. Nicht-Handeln des Staates kämpfen.

Seine geschlechterpolitisch reflektierte Perspektive auf den Staat führt Jessop ${ }^{25}$ zu einer umfänglichen Forschungsagenda. Für das hier fokussierte, exemplarische Untersuchungsfeld der Vergewaltigung in der Ehe steht von dieser erstens die Analyse der „maskulinistischen“ Überformung der politischen Repräsentation im Parteiensystem sowie der korporatistischen Interessengruppen und sozialen Bewegungen, also deren „Bemanntheit“ ${ }^{26}$ im Vordergrund. Zweitens interessiert die Architektur des Staates, d.h. die Kräfteverhältnisse zwischen den einzelnen Teilen der Staatsapparate (Exekutive, Legislative und Judikative), die Hierarchien der Ressorts sowie die Existenz informeller, parallel bestehenden Macht-Netzwerke, wohl wissend, dass der Anteil von Frauen umso geringer ist, je näher ein Ressort oder ein Netzwerk dem Kern des „repressiven Staatsapparates“ (Außenpolitik, innere Sicherheit, Finanzen, Wirtschaft) kommt. Und drittens interessiert der spezifische Einsatz staatlicher Interventionsmedien, konkret Zwangsgewalt, Geld, Recht und Wissen. Diese Interventionsmittel fundieren stets zugleich Prozesse vergeschlechtlichender Subjektkonstitution in Verschränkung mit den sozialen Platz anweisenden Kategorien von Hautfarbe, Klassen-, ethnischer und nationaler Zugehörigkeit, Alter und sexueller Orientierung. Hierbei handelt es sich um Prozesse der Fremdwahrnehmung, Selbstdeutung und Identitätsbildung, also der Subjektivierung, ${ }^{27}$ da der jeweilige Einsatz dieser Interventionsmedien eine geschlechtsspezifische Form der „Anrufung “ ${ }^{28}$ der Subjekte bedingt. Staatliche Politiken gestalten somit die Geschlechterverhältnisse nicht nur materiell, sondern bilden zugleich Orte und Knotenpunkte von Diskursen über zwei differente, auch in Bezug auf ihr sexuelles Begehren komplementär aufeinander bezogene Geschlechter mit jeweils spezifischen Funktionen, Rechten und Pflichten. Der Staat ist daher zugleich auch eine „Diskurs-

Jessop, Nationalstaat, Globalisierung, Gender, in: Kreisky/Sauer (Hrsg.), Geschlechterverhältnisse im Kontext politischer Transformation, Politische Vierteljahresschrift, Sonderheft 28/1997, S. 285.

26 Kreisky, Diskreter Maskulinismus. Über geschlechtsneutralen Schein politischer Idole, politischer Ideale und politischer Institutionen, in: dies./Sauer (Hrsg.), Das geheime Glossar der Politikwissenschaft. Geschlechtskritische Inspektion der Kategorien einer Disziplin, 1997, S. 161-213. 
arena",29 in der um die Hegemonie über Praktiken und Identitäten gefochten wird. In dieser Arena konstituieren sich nicht nur Subjektivitäten, also Selbstverhältnisse und Selbstwahrnehmungen, sondern zugleich von den Subjekten als möglich betrachtete, politische Strategien und Eingriffsmöglichkeiten. ${ }^{30}$

Dieser diskurstheoretische Zugang zu Staatlichkeit und deren Subjektivierungsmacht öffnet den analytischen Fokus dafür, dass und wie der Staat Frauen und Männer als untergeordnet und abhängig bzw. dominant und autonom „anruft“. ${ }^{31}$ Zugleich hat er somit Anteil an der Konfiguration „hegemonialer Männlichkeit“ als der Verkörperung der „momentan akzeptierten Antwort auf das Legitimitätsproblem des Patriarchats“, 32 auch wenn angesichts der „Staatsmacht", also aller gesellschaftlichen Kräfte, die im und durch den Staat agieren, die Dominanz der Männer sowie die Unterordnung der Frauen immer auch kontrolliert und in Grenzen gehalten werden muss. Eine Vermessung des „komplizierten Gitterwerks konfligierender Machtstrategien, -technologien und -diskurse ${ }^{\text {33 }}$ am Untersuchungsfeld der sexualisierten Gewalt gegen Frauen soll im nun folgenden Schritt mit Hilfe einer Skizze des in drei Etappen geführten Prozesses der Verrechtlichung von Vergewaltigung in der Ehe als Straftatbestand gegen die sexuelle Selbstbestimmung unternommen werden.

\section{Geschlechterpolitische Kämpfe um die Ent-Privatisierung sexueller Gewalt in der Ebe}

Ende 1970 diskutierten 31 mehrheitlich männliche Strafrechtsprofessoren in der „Sachverständigenanhörung“ zum Vierten Strafrechtsreformgesetz im deutschen Bundestagssonderausschuss ausführlich Argumente für und gegen eine Strafbarkeit ehelicher Vergewaltigung. ${ }^{34}$ In dieser ersten Etappe der Auseinandersetzungen definierten die zivilgesellschaftlichen Experten Vergewaltigung innerhalb der Ehe als „sexuelle Handlung“ im Rahmen langjähriger Partnerbeziehungen. Gegenüber den Rechtsexperten der Fraktionen plädierten sie mit Blick auf „Minderheiten“ (insbesondere Homosexuelle) und „Grenzfragen“ rechtsstaatlicher Regelungsbereiche, konkret die von staatlichen Interventionen frei zu haltenden sexuellen Aktivitäten des „mündigen Bürgers“, für einen Rückzug des Strafrechts aus der Privat- und Intimsphäre. Vor dem Hintergrund ihrer Argumentationen entschied sich der Rechtsausschuss des Bundestages mit einer Stimme Mehrheit gegen die Strafbarkeit der Vergewaltigung innerhalb der Ehe. Der im Anschluss von der sozial-liberalen Koalition der Legislative zur Abstimmung vorgelegte Regierungsentwurf sah nun sogar vor, auch unter $\ 178 \mathrm{StGB}$ („sexuelle Nötigung“) das Merkmal „außerehelich“ einzufügen. Unter der neuen Überschrift „Straftaten gegen die sexuelle Selbstbestimmung“ trat Ende 1973 das

29 Dackweiler, Staatliche Rechtspolitik als geschlechterpolitische Handlungs- und Diskursarena. Zum Verrechtlichungsprozess von Verwaltigung in der Ehe, in: dies./Schäfer (Hrsg.), Gewalt-Verhältnisse. Feministische Perspektiven auf Geschlecht und Gewalt, 2002, 107-131.

30 Demirovic/Pühl, Identitätspolitik und die Transformation von Staatlichkeit: Geschlechterverhältnisse und Staat als komplexe materielle Realität, in: Kreisky/Sauer (Hrsg.), Geschlechterverhältnisse im Kontext politischer Transformation, Politische Vierteljahresschrift, Sonderheft 28/1997, S. 231.

31 Für die im Umbruch befindliche keynesianisch-fordistischen Ära (wohlfahrts)staatlicher Politiken umfasst dieser Prozess der Subjektivierung die Anrufung der BürgerInnen als heterosexuell aufeinander verwiesene, deutsche (Ehe-)Frauen und (Ehe-)Männer in ihrer weiblichen Zuständigkeit für die Versorgungsökonomie und verstärkt auch als „Dazuverdienerinnen“ einerseits und den von „care“-Arbeit freigestellten männlichen Familienernährer und Familienvorstand andererseits; vgl. Dackweiler, Wohlfahrtsstaat: Institutionalisierte Regulierung und Transformation der Geschlechterverhältnisse, in: Beck/ Kortendiek (Hrsg.), Handbuch Frauen- und Geschlechterforschung. 2., 2008, S. 512-523.

32 Connell, Der gemachte Mann. Konstruktion und Krise von Männlichkeiten, 1999, S. 98.

33 Sauer, Die Asche des Souveräns. Staat und Demokratie in der Geschlechterdebatte, 2001, S. 162.

34 Das Strafrecht kodifzierte zu diesem Zeitpunkt unter dem Titel „Verbrechen und Vergehen wider die Sittlichkeit“ den Straftatbestand der "Vergewaltigung unter $\$ 177$ StGB als ,außerehelich“. 
Vierte Strafrechtsreformgesetz in Kraft, das unter den $\int \mathbb{S} 177$ und 178 StGB ehe-

liche sexuelle Gewalt ausklammerte.

Die zweite Etappe der Auseinandersetzungen eröffnete im Oktober 1983 ein Initiativantrag im Bundesrat zur Streichung des Wortes „außerehelich“ aus den \S 177 und 178 StGB durch das SPD-regierte Hamburg, dem sich die ebenfalls SPD-regierten Länder Bremen sowie Nordrhein-Westfalen anschlossen. Im Dezember desselben Jahres folgte ein von der Fraktion Die GRÜNEN in den Bundestag eingebrachter Entwurf für ein „Strafrechtsänderungsgesetz“, der sich auch mit Straftaten gegen das sexuelle Selbstbestimmungsrecht befasste, und nur eine Woche darauf präsentierte die Bundestagsfraktion der SPD einen Gesetzentwurf „zum besseren Schutz der Opfer von Sexualdelikten“. Die Gesetzesinitiativen wurden an die zuständigen Bundestagsausschüsse (Justiz, Jugend/Familie/Gesundheit und Inneres) zur Beratung überwiesen, die ein Sachverständigen-Hearing beschlossen, das jedoch erst 1986 vor dem Rechtsausschuss des Bundestages stattfand. Neben mehrheitlich männlichen Vertretern des Deutschen Richterbundes und des Anwaltvereins, der Bundesrechtsanwaltskammer, dem Bundeskriminalamt, dem Max-Planck-Institut für ausländisches und internationales Strafrecht und prominenten Strafrechtsprofessoren waren nun - im Unterschied zur Experten-Anhörung 1972 - auch Vertreterinnen der Evangelischen Aktionsgemeinschaft für Familienfragen, der Arbeitsgemeinschaft katholischer Verbände, der Arbeitsgemeinschaft Deutscher Frauen- und Kinderschutzhäuser, des Juristinnenbundes sowie die feministische Sozialwissenschaftlerin Ulrike Teubner und die Juristinnen Bärbel Wiegmann und Alexandra Goy geladen. Während alle weiblichen Sachverständigen für eine Strafbarkeit plädierten, vermochten die Pönalisierungsgegner kein „zahlenmäßig gravierendes Problem“ zu erkennen und gruppierten ihre Gegenargumente um die familienpolitische Achse des verfassungsrechtlich verankerten Schutzes von Ehe und Familie und deren Gefährdung durch eine strafrechtliche Intervention.

Im Sommer 1989 legten Die GRÜNEN im Kontext ihres „Antidiskriminierungsgesetzes“ noch einmal einen „Entwurf eines Gesetzes zur Änderung strafrechtlicher und strafprozessualer Regelungen bei Taten gegen die sexuelle Gewalt" vor. Der Entwurf, Ergebnis einer fünf Jahre dauernden Kontroverse zwischen Parteibasis, autonomen Frauen und der Bundestagsfraktion Die GRÜNEN, trug sowohl feministischen Argumentationen zur Reform der $\int \mathbb{S} 177$ und $178 \mathrm{StGB}$ als auch den Forderungen von AktivistInnen im Kampf für eine diskriminierungsfreie homosexuelle Lebensweise umfassend Rechnung: Er war geschlechtsneutral formuliert („eine Person“), verwandte einen inklusiven Gewaltbegriff („gegen ihren Willen“), differenzierte die Tathandlungen („anal, oral, vaginal penetriert oder in anderer Weise in ihren Körper eindringt oder hierzu Gegenstände benutzt“) und strich die Qualifizierung „außerehelich“ sowie den „minder schweren Fall“. 35

Erst die anfänglich dem Thema zögerlich gegenüberstehende, 1986 von Bundeskanzler Kohl bestellte erste Bundesfrauenministerin, Rita Süssmuth (CDU), verlieh - gegen den massiven Widerstand der männlichen Mitglieder der Fraktionen von CDU und CSU - der Reform der $\iint 177$ und 178 StGB eine zentrale frauenpolitische Bedeutung der Bundesregierung: Süssmuth erarbeitete Anfang 1988 in Zusammenarbeit mit dem F.D.P.-geführten Justizministerium einen Gesetzentwurf, der sich zu feministischen Forderungen (s.o.) an entscheidenden Punkten responsiv verhielt. Gleichwohl wollte Süssmuth im Falle ehelicher Vergewaltigung die Mindeststrafe auf ein Jahr senken, in minder schweren Fällen auf 
drei Monate, und der Ehefrau ein weiteres Mal mit Blick auf die „Aufrechterhaltung der bestehenden Bindung“ ein Widerspruchsrecht einräumen, das eine weitere Strafverfolgung des Ehemannes ausgeschlossen hätte. Die Ministerin scheiterte mit diesem Entwurf an der Phalanx der männlichen Unionspolitiker, die trotz der bereits 1986 vom Europäischen Parlament ergangenen Aufforderung an alle EU-Mitgliedstaaten, die Ungleichbehandlung von Ehefrauen im Falle sexualisierter Gewalt durch den Ehemann aufzuheben, am Ehegattenprivileg festhielten. Das Hauptargument der Strafbarkeitsgegner im christlichsozialliberalen Regierungslager konzentrierte sich Ende der 1980er Jahre nun darauf, dass bei einer Vergewaltigung innerhalb der Ehe der Frau keine „kriminologische Indikation “ für einen straffreien Schwangerschaftsabbruch erstellt werden dürfe, um Ehefrauen die „Erschleichung“ einer Indikation zu verstellen.

Anfang der 1990er Jahre lagen dem Deutschen Bundestag abermals drei Gesetzesentwürfe zur Änderung des Sexualstrafrechts zur Beschlussfassung vor und eröffneten die dritte Etappe des Diskussionsprozesses: ein Entwurf der SPDFraktion, einer des Bundesrates unter Federführung des Bundesministers der Justiz (F.D.P.) und einer der Abgeordneten Christina Schenk/ Bündnis 90/Die GRÜNEN. Daneben hatten die „Niedersächsische Kommission zur Reform des Strafrechts und des Strafverfahrensrechts“ und der Deutsche Juristinnenbund jeweils Reformvorschläge in der Öffentlichkeit präsentiert. Alle Entwürfe, die am Widerstand der CDU/CSU-Regierungsfraktionen scheiterten, sahen eine Streichung des Tatbestandsmerkmals „außerehelich“ vor; doch bis auf den Entwurf von Schenk/Bündnis 90/Die GRÜNEN sollten im Falle ehelicher Vergewaltigung Diversionsregeln ${ }^{36}$ greifen. Nach Verabschiedung des neu gefassten Abtreibungsparagraphen 218, der nun - erzwungen durch das Urteil des Bundesverfassungsgerichts - eine Fristenregelung mit „ergebnisoffener“ Zwangsbe-

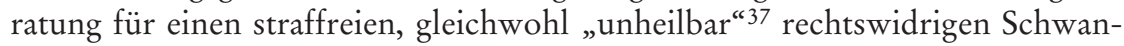
gerschaftsabbruch und die Kostenübernahme durch die betroffene Frau gesetzlich festschrieb, wiederbelebten im Frühjahr 1995 Abgeordnete der CDU die parlamentarische Diskussion zur Verrechtlichung ehelicher Vergewaltigung, diese nun erstmals nachdrücklich fordernd. Der dann im Frühjahr 1995 unter Federführung von Ministerin Leutheusser-Schnarrenberger (F.D.P.) im Justizministerium erarbeitete Regierungsentwurf bezog sich einmal mehr auf die frauenpolitische Forderung der Gleichbehandlung und stellte den Entwurf unter dem programmatischen Titel „Das Recht auf sexuelle Selbstbestimmung ist unteilbar“, obwohl auf Druck von CDU/CSU erneut eine Diversionsregel vorgesehen war.

Im September desselben Jahres einigten sich die Rechtsexperten von CDU/CSU und F.D.P. auf einen überarbeiteten Entwurf, der im Dezember 1995 im Rechtsausschuss zur Diskussion stand. Jener fasste Vergewaltigung und sexuelle Nötigung zu einem Einheitstatbestand zusammen ${ }^{38}$ und berücksichtigte neben körperlicher Gewalt auch die „Ausnutzung einer hilflosen Lage“ des Opfers. Wie schon im Entwurf von Süssmuth vorgesehen, hatten sich die Regierungsfraktionen darüber hinaus auf eine geschlechtsneutrale Bestimmung des Opfers geeinigt und räumten - im Rekurs auf die Aufrechterhaltung der ehelichen Bindung -

36 Unter dem strafverfahrensrechtlichen Begriff der Diversion wird die Unterlassung der Eröffnung eines richterlichen Strafprozesses verstanden, um das Delikt unter Absehung einer Strafverfolgung mit dem Ziel der Resozialisierung des Täters und der Entlastung der Gerichte von Bagatellverfahren zu erledigen.

37 Berghahn, Die neue Unübersichtlichkeit der Grenzüberschreitungen. Aktuelle Entwicklungen in der rechtlichen Regulierung des Privaten, in: Jurczyk/Oechsle (Hrsg.), Das Private neu denken. Erosionen, Ambivalenzen, Leistungen, 2008, S. 197.

38 „besonders schwere“ bzw. „minder schwere“ Fälle entsprechend dem Tatbestand des Eindringens in den Körper bzw. sexuelle Handlungen, die nicht mit dem Eindringen in den Körper verbunden sind. 
Ehefrauen ein „irreversibles Widerspruchsrecht“ ein. Auf Grund des Widerspruchsrechts des Opfers, das nun alle Oppositionsparteien kritisierten, scheiterte im Oktober 1996 auch dieser Gesetzesentwurf am Einspruch des Bundesrates, in welchem die SPD-regierten Länder die Mehrheit stellten. Abgelehnt wurde, so die Einschätzung der SPD-Frauenministerinnen aus zehn Bundesländern, ein „Zweiklassen-Strafrecht“, denn die Regierungsvorlage beabsichtigte, die Strafverfolgung des Täters für den gesamten Bereich der „häuslichen Gewalt“ unter den Vorbehalt zu stellen, dass das Opfer einer Vergewaltigung und/oder Misshandlung innerhalb der Ehe einer von ihr gestellten Strafanzeige nicht widerspricht beziehungsweise dass es dem Täter nicht gelingt, das Opfer zum Widerspruch zu erpressen. Die Ehefrau als Opfer sexueller Gewalt durch den Ehemann sollte im Unterschied zu den Opfern eines „Fremdtäters“ bzw. Partners einer nicht-staatlich sanktionierten Intimbeziehung die Rolle einer „Herrin des Verfahrens“ erhalten. Die Verantwortung der Tat somit an das Opfer delegierend, sollte das staatliche Gewaltmonopol in der Sphäre ehelicher Privatheit ein weiteres Mal zur „Aufrechterhaltung der ehelichen Bindung“ aufgehoben werden.

Nachdem es auch dieser Regierungskoalition nicht gelungen war, einen konsensfähigen Gesetzentwurf vorzulegen, fanden sich die weiblichen Abgeordneten des Bundestages - wie bereits beim $\ 218$ StGB im Einigungsvertrag - für einen Gruppenantrag über die Fraktionsgrenzen hinweg zusammen. Wortgleich mit der Koalitionsvorlage, jedoch ohne Widerspruchsregelung ausgestaltet, wurde dieser Gruppenantrag im Mai 1997 von der Mehrheit der 644 Abgeordneten im Deutschen Bundestag in „freier Gewissensentscheidung“, d.h. ohne Fraktionszwang, angenommen.

\section{Transformationen „strategischer Selektivitäten“ und Verschiebungen „strukturorientierter strategischer Kalkulationen" aus feministischer Perspektive}

Die in der Bundesrepublik Deutschland drei Jahrzehnte umspannenden geschlechterpolitischen Kontroversen über eine Aufhebung des „Ehegattenprivilegs" wurden in Expertenkommissionen, Justiz, Legislative, Exekutive sowie von den hier seit den späten 1970er Jahren institutionalisierten „Femokratinnen“, in Parteien, Verbänden, Kirchen, Fach-, Tages- und politischen Wochenzeitschriften, von WissenschaftlerInnen sowie Aktivistinnen der Frauenbewegung, Mitarbeiterinnen von autonomen Projekten (Frauenhäusern, -beratungsstellen und -notrufen) sowie von Frauen-NGOs geführt. In diesen Auseinandersetzungen rangen jeweils strukturorientierte, strategisch kalkulierende AkteurInnen um Definitionsmacht darüber, was von universalem, gesamtgesellschaftlichen Interesse, mithin als politisch und daher als Gegenstand öffentlicher Intervention, also als „Staatsaufgabe“ gelten soll. Somit ging es um die Anfechtung, Verteidigung und Neujustierung des in den Strukturen des Ensembles der diskursiven Arenen und Institutionen geronnenen Konsenses über die staatliche Nicht-Einmischung in gewaltförmig durchgesetzte, ehemännliche Dominanz- und Verfügungsansprüche. Es handelte sich aber zugleich auch um Kämpfe um Verhandlungsmacht innerhalb der Staatsapparate sowie um Zugang zu deren Machtressourcen, also um Verschiebungen der geschlechtsspezifischen strategischen Selektivitäten dieser Apparate, um deren Abwehr und um neue Kompromissbildungen. Zuletzt können diese Auseinandersetzungen aufgeschlüsselt werden als Kämpfe um vergeschlechtlichende Anrufungen im Kontext von (Hetero-)Sexualität und Gewalt: Zur Verhandlung stand konkurrierendes Wissen darüber, wer das „verletzungs- 
offene“ und wer das „verletzungsmächtige“ Geschlecht in Bezug auf körperliche Integrität und sexuelle Selbstbestimmung ist und wem sodann unter Bezugnahme auf diesen Objekt- bzw. Subjektstatus Anspruch auf staatlichen Schutz vor Gewalt im sozialen Nahbereich zukommen soll und muss. ${ }^{39}$

Der Ausgang der Sexualstrafrechtsreform 1973 wurde maßgeblich beeinflusst durch die Gutachtermeinung sich liberal definierender Strafrechtsprofessoren und ihres Interesses, „sexuelle Praktiken“ zu entkriminalisieren und den Staat aus der als privat definierten Sphäre ehelicher Beziehungen zurückzudrängen. Eingelassen in einen sexualpolitischen Liberalisierungsdiskurs, der die Grenzen zwischen „staatsfreier" Privatheit und staatlich regulierter Öffentlichkeit vertiefte bzw. neu zog, verschwand in dieser ersten Etappe für die männlichen Rechtswissenschaftler und Abgeordneten der sozial-liberalen Regierungsparteien die strafrechtliche Relevanz des gewaltförmigen Angriffs des Ehemannes auf das sexuelle Selbstbestimmungsrecht der Ehefrau hinter dem politischem Interesse, den Staat aus der Regulierung „komplexer Lebensverhältnisse“ herauszuhalten und die staatlichen Möglichkeiten der Einschränkung der „Pluralität der Lebensformen und Erlebnismöglichkeiten " 40 zu begrenzen. Hierbei bildeten die reformpolitischen Argumente des Schutzes der Privatsphäre von Individuen in Ehe und Familie vor dem Staat eine Allianz mit dem zentralen konservativen politischen Ziel des Schutzes, sprich der Privilegierung von Ehe- und Familie als einzig legitimer Lebens- und Reproduktionsform, durch den Staat, ungeachtet des Ausmaßes der hier von Männern gegen Frauen ausgeübten Gewalt. Im Unterschied zu den folgenden Etappen der Diskussion in den 1980er und 1990er Jahren fand neben der juristischen eine sozialwissenschaftlich Politik beratende Expertise, welche die macht- und herrschaftstheoretischen Dimensionen von (geschlechtsbezogener) Gewalt hätte thematisieren können, keinen entscheidungsrelevanten Eingang in die Legislative und Exekutive. Zugleich gilt es festzuhalten, dass zu diesem Zeitpunkt weder nationale oder internationale empirische Studien zur Prävalenz und Inzidenz von Vergewaltigung in der Ehe, noch feministisch orientierte Analysen der sexuellen Gewalt gegen Frauen im sozialen Nahbereich vorlagen und auch die noch junge Neue Frauenbewegung dieses für sie in den 1980er und 1990er Jahren zentralen Anliegen noch nicht öffentlichkeitswirksam aufgegriffen hatte. Ebenso wenig existierte der Einflusskanal institutionalisierter Frauenpolitik auf die Staatsapparate, um einen wirkmächtigen Versuch des Einspruchs gegen die, von der Strafrechtsreform im Horizont einer „emanzipatorischen“ und „anti-autoritären“ Rechtspolitik normierten Verweigerung des staatlichen Schutzes und der Fürsorge gegenüber Ehefrauen im Falle der sexuellen Gewalt durch den Ehemann zu unternehmen.

Die Effekte dieser geschlechtsspezifischen strategischen Selektivitäten zeigen sich überdeutlich mit Blick auf den Beginn der zweiten Etappe 1983, denn der Initiativantrag aus Hamburg, unter Federführung seiner Justizsenatorin, wurde angestoßen durch eine von der „Leitstelle für die Gleichberechtigung der Frau“ finanzierte Gewalt-Studie ${ }^{41}$ und dem hier angesichts des Ausmaßes und der Folgen von (sexueller) Gewalt gegen Ehefrauen aufgezeigten politischen Handlungsbedarf. So erwiesen sich zum einen die seit den späten 1970er Jahren in den

39 Dackweiler, Rechtspolitische Konstruktionen sexueller Verletzungsoffenheit und Verletzungsmächtigkeit: Zur Verrechtlichung von Vergewaltigung in der Ehe in der Schweiz und der Bundesrepublik Deutschland, in: Koher/Pühl (Hrsg.), Gewalt und Geschlecht. Konstruktionen, Positionen, Praxen, 2003, S. 62.

40 Jäger, Entkriminalisierungspolitik im Sexualstrafrecht, in: ders./Schorsch (Hrsg.), Sexualwissenschaft und Strafrecht, 1987, S. 2.

41 Sadrozinski/ Schmidt-Bott, Geprügelt, getreten, vergewaltigt. Studie der Leitstelle Gleichstellung der Frau, Staatliche Pressestelle Hamburg: Berichte und Dokumente, Nr. 619/1980. 
Ländern und Kommunen geschaffenen Gleichstellungsinstitutionen als ideelle und finanzielle Ressource bei der Öffnung des staatsbürokratischen und parlamentarischen Entscheidungsraums für feministische Forderungen. Zum anderen war die Schaffung des vierten Aufgabengebiets „Frauen“ im Bundesministerium für Jugend, Familie und Gesundheit im Jahr 1986, das auf Grund der definierten „Querschnittsaufgabe“ mit erweiterten Rechten ausgestattet wurde, von entscheidender Bedeutung für die Dynamik und Argumentationen der Auseinandersetzungen. Zugleich positionierte sich die SPD, ebenso wie die 1983 erst wenige Monate im Bundestag vertretene Partei Die GRÜNEN, mit ihren Anträgen in der Rolle der Opposition im Bundestag im neuen Politikfeld der „Frauenpolitik“ unter der christlichsozial-liberalen Regierung von Kanzler Helmut Kohl und dessen Programmatik einer „konservativen Wende“. Insbesondere Die GRÜNEN - Sammelbecken nicht nur von AktivistInnen der Friedens- und Ökologiebewegung, sondern auch der Neuen Frauen- sowie der Homosexuellenbewegung - nahmen Bezug auf nun vorliegende Erkenntnisse feministisch informierter Studien zum Ausmaß, einzelnen Formen von (sexueller) Gewalt sowie den Auswirkungen auf die Opfer. ${ }^{42}$ Diese empirischen Studien widerlegten die hegemoniale Annahme, dass „Fremdtäter“, ebenso wie eheliche Vergewaltiger, aus sexuellen Motiven bzw. auf Grund ihres unkontrollierten „Sexualtriebs“ handelten. Demgegenüber fokussierten die Autorinnen ${ }^{43}$ die geschlechtsbezogene Gewaltaktion des Täters zur Durchsetzung seines Dominanz-, Verfügungsund Machtanspruchs in einer unterstellten, natürlichen Ordnung der Geschlechter und verwiesen aus einer herrschaftstheoretischen Perspektive auf die Billigung dieser Gewalt durch eine patriarchale Polizei und Justiz, deren Praktiken bzw. Rechtsnormen und Rechtsprechung hegemoniale Männlichkeit sicherten und (sexualisierte) Gewalt nicht als Normverletzung, sondern „Normverlängerung “44 erscheinen ließen.

Vor diesem Hintergrund forderten Sozialwissenschaftlerinnen, Juristinnen und Mitarbeiterinnen der unterdessen im gesamten Bundesgebiet entstandenen Schutz- und Beratungsprojekte für von Gewalt betroffene Frauen via ihres Einflusses auf kommunale Gleichstellungsbeauftragte, Femokratinnen in der Exekutive des Bundes und der Länder sowie auf die parlamentarische Opposition, wohlfahrtsstaatliche Verbände und Medien, nicht mehr nur die Ausweitung der \S 177 und 178 StGB auch auf eheliche Vergewaltigung und Nötigung, sondern darüber hinaus eine Neudefinition des Gewaltbegriffs, eine Differenzierung der Tathandlungen und die Streichung des „minder schweren Falls“. Hierbei konkurrierten sie in den verschiedenen Diskursarenen um den Einfluss ihres Wissens über (Hetero-)Sexualität, Macht und Gewalt im Geschlechterverhältnis mit angesehenen Vertretern einer links-liberalen Kriminalsoziologie und Sexualforschung, die den geschlechtsbezogenen Charakter der sexuellen Gewalt innerhalb der Ehe zwar nicht mehr verneinten, jedoch im Rahmen der Auseinandersetzung um eine Strafbarkeit von ehelicher Vergewaltigung an der Position festhielten, dass es sich bei Vergewaltigung um das „private Konfliktfeld“45 der Sexualität von Männern und Frauen handele.

43 Müller, Privatheit als Ort geschlechtsbezogener Gewalt, in: Jurczyk/Oechsle (Hrsg.), Das Private neu denken. Erosionen, Ambivalenzen, Leistungen, 2008, S. $228 \mathrm{f}$.

44 Hagemann-White, Strategien gegen Gewalt im Geschlechterverhältnis. Bestandsanalysen und Perspektiven, 1992 , S. 10.

45 Schorsch, Vergewaltigung in der Ehe, in: Jäger/Schorsch (Hrsg.), Sexualwissenschaft und Strafrecht, 1987, S. 116. 
Diese Konfliktlinie zwischen potentiellen BündnispartnerInnen verweist zugleich exemplarisch auf die Bedeutung von Allianzstrukturen zwischen zivilgesellschaftlichen Akteuren und jenen der „politischen Gesellschaft“. Konkret zwischen einerseits libertär sich verstehenden Hochschulprofessoren als Vertreter einer nicht repressiven, „gewaltfreien Kriminalpolitik“ und andererseits christlich-konservativen Familienpolitikern, die - angetrieben von unterschiedlichen Interessen - eine wirkmächtige Verharmlosung und Verkennung sexueller Gewalt innerhalb der Ehe im Rekurs auf das Argument der Sicherung der von staatlicher Interventionen frei zu haltenden „Privatsphäre“ und der durch staatliche Intervention nicht zu behindernden „Aufrechterhaltung der ehelichen Bindung“ betrieben. Zugleich affirmierte auch diese Allianz die symbolische Ordnung der heterosexuellen Zweigeschlechtlichkeit, in der jeweils ein sexuell verletzungsoffenes Geschlecht, konkret das weibliche, und ein sexuell verletzungsmächtiges Geschlecht, konkret das männliche Geschlecht, „angerufen“ wurde. Demgegenüber sollte der geschlechtsneutral formulierte Reformvorschlag dazu verhelfen, sowohl Ehefrauen als Opfer sexueller Gewalt in das Sexualstrafrecht $\mathrm{zu}$ inkludieren als auch die Einsicht zu institutionalisieren, dass jede Person unabhängig von ihrem Geschlecht und von sexuierten Körperöffnungen - sexuell verletzt werden kann und Verletzungsmächtigkeit ebenfalls nicht von sexuierten Körperteilen abhängig ist.

In der dritten Etappe verschoben sich die „strukturorientierten strategischen Kalkulationen“ und in ihrer Dialektik zugleich die „strategischen Selektivitäten“ des integralen Staats nachhaltig aufgrund des von der UN und den transnationalen Frauenbewegungen seit Beginn der 1990er Jahre geführten Diskurses der Frauenmenschenrechte. So gilt es, die Bemühungen der CDU-Rechtsexperten für eine Strafbarkeit von Vergewaltigung innerhalb der Ehe im Frühjahr 1995, nach über zehn Jahren konservativ-patriarchaler Blockadepolitik, vor diesem Hintergrund zu verstehen. Vor allem die Vierte Weltfrauenkonferenz im Sommer 1995 in Beijing brachte Frauenmenschenrechte erneut weltweit auf die mediale Tagesordnung und verschob die Kräfteverhältnisse zur Durchsetzung der Forderung einer Pönalisierung von Vergewaltigung in der Ehe, wurde doch die seit Ende der 1960er Jahre kanonisierte Argumentationsfigur gebotener staatlicher Nicht-Einmischung in die eheliche Privat- und Intimsphäre delegitimiert: Für die bundesdeutsche Diskussion um eine Strafbarkeit von Vergewaltigung in der Ehe bedeutete die „neue“ Verantwortung des Staates für den Menschenrechtsschutz von Frauen, dass das in Art. 2 Abs. 2 GG definierte Grundrecht auf Leben und körperliche Unversehrtheit nicht länger nur als Schutz vor staatlichen Eingriffen interpretiert werden darf, der den Staat darauf verpflichtet, Eingriffe in die Privatsphäre zu unterlassen. Vielmehr rückt nun die Schutzpflicht des Staates gegenüber den Grundrechten von (Ehe-)Frauen im Falle der sexuellen Gewalt im sozialen Nahbereich in den Fokus staatlichen Handelns. Diese neue Diskurs- und Kräftekonstellation strukturierte den Kontext und die Strategien aller AkteurInnen im Rechtsausschuss zur Diskussion des Gesetzentwurfs der christlichsozial-liberalen Regierungskoalition im Winter 1995: Feministisch orientierte VertreterInnen situierten sich mit ihrer Forderung nach einer Pönalisierung nun offensiv im international hegemonialen Diskurs der Frauenmenschenrechte, so etwa die Vertreterin der Frauenmenschenrechtsorganisation „terre des femmes“ in der Sachverständigenanhörung, während dessen Regierungsparteien und Exekutive sich in diesem Bezugsrahmen unter globaler Beobachtung durch die Vereinten Nationen sehen durften.

In dieser Diskurs- und Kräftekonstellation lässt sich auch die Genese und Ausgestaltung des fünfJahre späten/in Kraft getretenen „Gewaltschutzgesetzes“ 
(GewSchG) rekonstruieren, mit welchem die erste rot-grüne Bundesregierung in der 14. Legislaturperiode intendierte, den zivilgerichtlichen präventiven Schutz vor Gewalttaten im „häuslichen Bereich“ sowie bei Nachstellungen zu verbessern. In enger Anlehnung an das in Österreich bereits seit 1997 erprobte „Bundesgesetz zum Schutz vor Gewalt in der Familie“, das unter maßgeblicher Beteiligung der österreichischen Frauenbewegung und Frauenorganisationen im Horizont der Frauenmenschenrechte entstand und sich zum anerkannten „bestpractice“-Modell in Westeuropa im Kampf gegen Gewalt gegen Frauen entwickelte, ${ }^{46}$ wird das GewSchG flankiert von entsprechenden Paragraphen der Polizeigesetze der Bundesländer. Diese sind gedacht als „Bindeglied“ 47 zwischen einerseits dem zivilrechtlichen Schutz der Opfer, konkret amtsgerichtlich zu beantragende Schutzanordnungen, Zuweisung der Wohnung, Schadensersatz und Schmerzensgeld und - so vorhanden - alleiniges Sorgerecht über die Kinder sowie die Aussetzung oder Beschränkung des Umgangsrechts und andererseits dem unmittelbaren staatlichen Eingreifen durch das Strafrecht, d.h. neben der Weitergabe von Informationen zu Beratungsangeboten die Anzeigenaufnahme und deren Weiterleitung an die Amts- oder Staatsanwaltschaft sowie die Wegweisung bzw. in Gewahrsamnahme des Täters durch die Polizei. Auch mit dieser Kodifizierung (re)definierte der bundesdeutsche Staat nunmehr seit einer Dekade seine Aufgabe in Bezug auf Gewalt im Geschlechterverhältnis, konkret Opferschutz und die eindeutige Ansage gegenüber den Tätern: „Gewalt wird vom Staat nicht toleriert, auch dann nicht, wenn sie sich in den eigenen vier Wänden vollzieht . 48

\section{Ambivalente Erfolge - widersprüchliche Entwicklungen: ein Fazit}

Warum nach über 25 Jahren der Kämpfe und Debatten im „integralen Staat“ über eine Strafbarkeit ehelicher Vergewaltigung das Finale nicht mehr von polarisierenden und kompromisslos sich gegenüberstehenden Positionen geprägt war, sondern in den Augen der Juristin Monika Frommel ${ }^{49}$ zuletzt als eine Reform „von oben“ in Erscheinung trat und die Verabschiedung des GewSchG ohne politischen Widerstand von statten gehen konnte, lässt sich - wie in Kapitel 5 entfaltet - mit Hilfe der Analyse der Transformationen geschlechtsspezifisch strategisch-selektiver Strukturen sowie der mit diesen in Wechselwirkung stehenden Verschiebungen der strukturorientierten strategischen Kalkulationen vergeschlechtlichter Subjekte nachvollziehen. Es gelang, die feministische Forderung nach sexueller Selbstbestimmung und der Wahrung des Grundrechts von Ehefrauen auf körperliche und psychische Integrität in das staatliche institutionelle Ensemble einzufügen, insbesondere auf Grund der Erosion seiner maskulinistischen Überformung, aber auch ausgelöst durch die aufbrechende Männerdominanz in den Macht-Netzwerken der Zivilgesellschaft einerseits und der internationalen Hegemonie des Diskurses der Frauenmenschenrechte andererseits. Die hierüber sich öffnenden Einflusskanäle verdichteten die Kräfteverhältnisse, die den Staat durchziehen, auf eine neue, emanzipatorische geschlechterpolitische

Dackweiler, „Wir sind der Nukleus der globalen Zivilgesellschaft“ - Zusammenspiel und Wechselverhältnis der internationalen und nationalen Frauenbewegungen am Beispiel des österreichischen Gewaltschutzgesetzes, in: Lenz/Mae/Klose (Hrsg.), Frauenbewegungen weltweit, 2000, S.167-198.

47 Eichler et al., Kooperationsformen und -strukturen von Runden Tischen / Arbeitskreisen zum Abbau Häuslicher Gewalt in NRW, hrsgg. v. Ministerium für Gesundheit, Soziales, Frauen und Familie NRW, S. 17.

48 BMFSFJ/BMJ, Mehr Schutz bei häuslicher Gewalt. Information zum Gewaltschutzgesetz. 3. Aufl. 2010, S. 3 .

49 Frommel, Zaghafte Versuche einer Reform der sexuellen Gewaitdelikte, KJ 1996, 168. 
Interessen inkludierende Weise, die zurück wirken auf die als möglich oder eben nicht mehr möglich betrachteten politischen Strategien von (kollektiven) AkteurInnen in der politischen und der Zivilgesellschaft.

Und doch komme ich aus feministischer Perspektive zu einer ambivalenten Bewertung der „Erfolgsgeschichte“ des errungenen Konsenses über die neue Staatsaufgabe, Egalität und (sexuelle) Gewaltfreiheit auch in den Intimbeziehungen von (Ehe)PartnerInnen zu normieren und zu sanktionieren. So gilt es erstens festzuhalten, dass hinsichtlich der hier exemplarisch analysierten Dimension der sexuellen Gewalt in der Ehe im Anschluss an das Inkrafttreten der Sexualstrafrechtsreform eine diskursive Friedhofsruhe eingetreten ist: International vernetzt befassen sich ForscherInnen mit dem Thema „häuslicher Gewalt“, sprich Misshandlung mit Blick auf Ursachen, Prävention, Täterarbeit und Betroffenheit von im Haushalt lebenden Kindern; sodann seit dessen Inkrafttreten 2002 mit der Evaluierung und Weiterentwicklung der Umsetzung des „Gewaltschutzgesetzes“, welches jedoch als Gewaltform die Verletzung der „sexuellen Selbstbestimmung“ nicht explizit benennt, ${ }^{50}$ und zuletzt - aus gegebenem Anlass - vermehrt mit dem Problem der sexuellen Übergriffe auf Kinder und Jugendliche in Heimen, Internaten und kirchlichen Einrichtungen. Demgegenüber richten bundesweit „kaum noch WissenschaftlerInnen ihren Fokus auf sexuelle Gewalt gegen erwachsene Frauen ", 51 obwohl die Dunkelfeldforschung bestätigt, dass jede siebte Frau mindestens einmal in ihrem Leben Vergewaltigung oder schwerer sexuelle Nötigung erlebt, nur fünf Prozent der Straftaten zur Anzeige gelangen und es nur bei 14,6 Prozent der erfassten Anzeigen im Jahr 2008 zu einer Verurteilung kam. ${ }^{52}$ Es muss also durchaus davon die Rede sein, dass Frauen als Opfer dieser Straftaten gegen die sexuelle Selbstbestimmung auch weiterhin in einer „Gerechtigkeitslücke“ leben. Inmitten des (globalen) Konsenses über die staatliche Verantwortung gegenüber geschlechtsbezogener Gewalt gilt es, eine erklärungsbedürftige politische Stille auch in der feministischen Diskussion über die in den bestehenden gesellschaftlichen Verhältnissen aufzusuchenden Ursachen sexueller Gewalt im sozialen Nahraum zu konstatieren, während dessen eine pathologisierende und erneut individualisierende medizinisch-psychologische Rede über die traumatisierenden Folgen dieser erlittenen Gewalt für die Opfer Deutungshoheit erlangt hat. ${ }^{53}$

Zweitens ist in diesen (globalen) Konsens ein widersprüchlicher Diskurs der Schutzbedürftigkeit und der Autonomie von Frauen eingeschrieben, die weiterhin die überwiegende Mehrheit der Opfer (sexueller) Gewalt innerhalb und außerhalb der Ehe stellen. ${ }^{54}$ Schutz und Unterstützung zu gewähren ist nun zwar eine öffentliche Angelegenheit, also eine des Staates, und nicht mehr die des „pater familias“. Zugleich werden „schutzbedürftige“, weil sexueller Gewalt ausgesetzte Frauen aber auch als „aktive Rechtssubjekte“ angerufen und sollen als sol-

50 Vgl. $\$ 1$ Abs. 1 sowie Abs. 2 des Gesetzes zum zivilrechtlichen Schutz vor Gewalttaten und Nachstellungen (Gewaltschutzgesetz - GewSchG), BGBl. I, 3513.

51 Grieger, Streitsache Sexualdelikte - Frauen in der Gerechtigkeitslücke, Streit. Feministische Rechtszeitschrift, H. 2/2011, 92.

52 Ebd., 93.

53 Landesarbeitsgemeinschaft der autonomen Notrufe in Rheinland-Pfalz, Traumatisierung durch sexualisierte Gewalt. Die Arbeit der Frauennotrufe im Kontext von Traumaforschung und gesellschaftlichen Mythen zu Vergewaltigung, Streit. Feministische Rechtszeitschrift, 1/2010, 8-10.

54 Vgl. auch Wetzels/Pfeiffer, Sexuelle Gewalt gegen Frauen im öffentlichen und privaten Raum. Ergebnisse der KFN-Opferbefragung 1992, 1995; Schröttle, Gewalt in Paarbeziehungen. Eine sekundäranalytische Auswertung zur Differenzierung von Schweregraden, Mustern, Risikofaktoren und Unterstützung nach erlebter Gewalt im Auftrag des BMFSFJ. Kurzfassung, 2008. 
che selbstbestimmt und ihre eigenen Risiken verwaltend handeln. ${ }^{55}$ Dies bringt sie innerhalb der fortbestehenden patriarchalen symbolischen Ordnung der Geschlechter und der in dieser hervorgebrachten vielfältigen und widersprüchlichen Selbstwahrnehmungen und Fremdzuschreibungen potentiell in mit einander konkurrierende und sich tendenziell ausschließende Subjektpositionen, konkret in die Position des autonomen, weiblich verkörpertes (Rechts-)Subjekts zur einen und zur anderen Seite der „loyalen (Ehe-)Frau“ und „guten Mutter“, die Bedürfnisse und Interessen zu Gunsten der Anderen zurückstellt. ${ }^{56}$ Auch dem zivilrechtlich angesiedelten Opferschutz des Gewaltschutzgesetzes in Verbindung mit der bundesländerspezifisch ausgestalteten polizeirechtlichen Wegweisung sowie strafrechtlichen Verfolgung der Täter weist diese Antinomie für die von Gewalt betroffenen Frauen auf: Einerseits entzieht sich - am weitestgehenden im nordrhein-westfälischen Polizeigesetz - das polizeiliche Eingreifen im Sinne der „Gefahrenabwehr und Strafverfolgung“ dem Willen des Gewaltopfers. Andererseits muss die von Gewalt betroffene Frau die zivilrechtlichen Schritte unverzüglich selbst einleiten, um auf gerichtlichem Weg als „vorbeugend“ definierte Schutzanordnungen zu erlangen.

Zugleich haben auch die ehemals ausschließlich am Komm- und Autonomieprinzip orientierten (feministischen) Beratungs- und Unterstützungsangebote für betroffene Frauen im Zuge der Re-Definition der Staatsaufgabe bei geschlechtsbezogener Gewalt und der in diesem Kontext geschaffenen Kooperationen und Vernetzungen aller mit von Gewalt im sozialen Nahraum betroffener Frauen und deren Kindern befassten Institutionen und Einrichtungen ihr Handeln unter dem Titel „Interventionsprojekte“ verändert bzw. verändern müssen: Dieses ist nicht mehr uneingeschränkt ausgerichtet an den Leitideen der Freiwilligkeit und Selbstbestimmung der Gewaltbetroffenen bezüglich Beratung, Unterstützung und Begleitung durch professionalisierte Einrichtungen. Ausgehend von Annahmen zu den eingeschränkten Ressourcen sowie bestehender Hilflosigkeit der Betroffenen in einer Krisensituation wird nunmehr auch ein „Pro-Aktiv-Ansatz“ verfolgt. In dessen Rahmen nehmen „Interventionsprojekte", benachrichtigt durch die Polizei nach deren Eingreifen, zeitnah Kontakt (telefonisch oder mittels „Hausbesuchen“) mit der Frau auf bzw. sind zu dieser Kontaktaufnahme verpflichtet und zwar - je nach Ausgestaltung des Polizeigesetzes - mit oder auch ohne ausdrückliche Zustimmung des Gewaltopfers. Somit konstituiert das neue Verständnis von Opferschutz und Gefahrenabwehr für jene eine widersprüchliche Zone zwischen Freiwilligkeit und „Zwangsberatung“57 und versetzt sie in ein ambigues Zugleich als Bevormundungsobjekt und Kontrollsubjekt im Horizont einer Sicherungsstrategie gegen eine vermutete „Autonomiefalle“. 58

Diese Antinomien des aktiven Rechtssubjekts einerseits und zwangsberatungsbedürftigen Schutzobjekts andererseits gilt es, aus einer feministisch informierten, staatstheoretischen Perspektive noch genauer als Teil der Widersprüche des

Marugàn/Vega, Feministische Praxis und Gouvernementalität. Das Regieren der Gewalt gegen Frauen, in: Pieper et al. (Hrsg.), Gouvernementalität. Ein sozialwissenschaftliches Konzept in Anschluss an Foucault, 2003, S. 107.

56 Merry, Rights Talk and the Experience of Law: „Implementing Women’s Human Rights to Protection from Violence“, in: Lookwood (Hrsg.), Women's Rights. A Human Rights Quarterly Reader, 2006, S. $393 \mathrm{ff}$.

57 Heidrich/Rohleder, Soziale Arbeit und Häusliche Gewalt. Ein Arbeitsfeld im Umbruch, in: Hasenjürgen/Rohleder (Hrsg.), Geschlecht im sozialen Kontext, 2005, S. $219 \mathrm{ff}$.

58 Diese würde als „Folge einer Überforderung des Opfers dem Gewalttäter in die Hände spielen“, vgl. Dearing, Das österreichische Gewaltschutzgesetz als Kern einer umfassenden Reform der Reaktion auf Gewalt in der Privatsphäre unter besonderer Berücksichtigung der Funktion der Sicherheitsexekutive, in: Dearing/Haller (Hrsg.), Das Österreichische Gewaltschutzgesetz, Wien, 2000, S. 93. 
sich aus dem fordistisch-keyneseanischen (Wohlfahrts-)Staat herausschälenden, neoliberal orientierten „schumpeteranischen Wettbewerbsstaats“ auszuleuchten. Zugleich muss der errungene Konsens über die neue Staatsaufgabe angesichts der im Kontext eines „aktivierenden Sozialstaats“ vorgenommenen Änderungen der Sozialgesetze, ${ }^{59}$ die den materiellen Schutz von Frauen, insbesondere von Asylsuchenden und jenen mit Aufenthaltsstatus, vor (sexueller) Gewalt wieder einschränken ${ }^{60}$ und erneut hierarchische und von ökonomischer Abhängigkeit gekennzeichnete Geschlechter- in Überschneidung mit anderen herrschaftsförmigen Sozialverhältnissen re-stabilisieren, auch weiterhin als ebenso unabgeschlossen wie angefochten betrachtet werden.

\section{Menschenrechtsförderung}

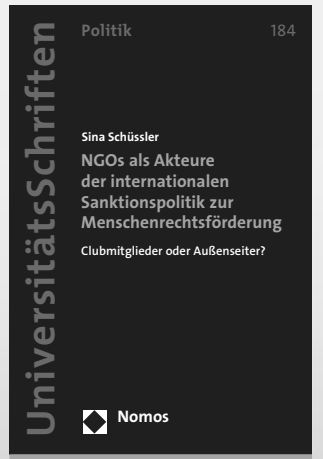

Weitere Informationen: www.nomos-shop.de/14425

NGOs als Akteure der internationalen Sanktionspolitik zur Menschenrechtsförderung

Clubmitglieder oder Außenseiter?

Von Dr. Sina Schüssler, M.A.

2012, 298 S., brosch., 49,-€

ISBN 978-3-8329-7255-4

(Nomos Universitätsschriften Politik, Bd. 184)

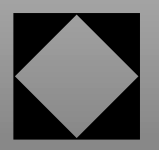

\section{Nomos} kungen von Hartz IV, 2010.

60 Vgl. Frauenkoordinierung. Zentrale Informationsstelle Autonomer Frauenhäuser, Positionspapier zur verlässlichen Absicherung der Hilfsangebote (insbesondere Schutz- und Unterstützungsangebote) bei häuslicher und sexualisierter Gewalt durch eine bundesweit verbindliche Regelung, 2008 (www.frauenhauskoordinierung.de, abgerufen am 18.12.2011). 